\title{
Self-efficacy and self-rated oral health among pregnant aboriginal Australian women
}

\author{
Lisa M Jamieson ${ }^{1 *}$, Eleanor J Parker ${ }^{1}$, Kaye F Roberts-Thomson ${ }^{1}$, Herenia P Lawrence ${ }^{3}$ and John Broughton ${ }^{2}$
}

\begin{abstract}
Background: Self-efficacy plays an important role in oral health-related behaviours. There is little known about associations between self-efficacy and subjective oral health among populations at heightened risk of dental disease. This study aimed to determine if low self-efficacy was associated with poor self-rated oral health after adjusting for confounding among a convenience sample of pregnant women.

Methods: We used self-reported data from 446 Australian women pregnant with an Aboriginal child (age range 14-43 years) to evaluate self-rated oral health, self-efficacy and socio-demographic, psychosocial, social cognitive and risk factors. Hierarchical entry of explanatory variables into logistic regression models estimated prevalence odds ratios (POR) and 95\% confidence intervals $(95 \% \mathrm{Cl})$ for fair or poor self-rated oral health.

Results: In an unadjusted model, those with low self-efficacy had 2.40 times the odds of rating their oral health as 'fair' or 'poor' (95\% Cl 1.54-3.74). Addition of socio-demographic factors attenuated the effect of low self-efficacy on poor self-rated oral health by 10 percent (POR 2.19,95\% Cl 1.37-3.51). Addition of the psychosocial factors attenuated the odds by 17 percent (POR 2.07, 95\% Cl 1.28-3.36), while addition of the social cognitive variable fatalism increased the odds by 1 percent (POR 2.42, 95\% Cl 1.55-3.78). Inclusion of the behavioural risk factor 'not brushing previous day' attenuated the odds by 15 percent (POR 2.11,95\%Cl 1.32-3.36). In the final model, which included all covariates, the odds were attenuated by 32 percent (POR 1.80, 95\% Cl 1.05, 3.08).
\end{abstract}

Conclusions: Low self-efficacy persisted as a risk indicator for poor self-rated oral health after adjusting for confounding among this vulnerable population.

\section{Background}

Self-efficacy has been described as one's confidence in his or her ability to behave in ways to produce a desirable outcome [1]. In the dental setting, relatively little research exists on self-efficacy. Klepac et al. [2] presented evidence that associated dental anxiety with low self-efficacy regarding ability to tolerate tooth pain. Similarly, Kent and Gibbons [3] demonstrated that those who are more anxious are less confident about their ability to control their fear-related emotions regarding dentistry. Low dental self-efficacy of carers was associated with higher caries levels among Head Start children in the United States [4], while parental self-efficacy was found to be the strongest predictor of children's brushing habits among pre-schoolers [5]. In a population

\footnotetext{
* Correspondence: lisa.jamieson@adelaide.edu.au

'Australian Research Centre for Population Oral Health, University of

Adelaide, Adelaide, SA 5005, Australia

Full list of author information is available at the end of the article
}

sample of low-income African Americans, Finlayson and colleagues [6] reported that maternal self-efficacy was a predictor of child brushing.

Bandura [1] referred to four sources of self-efficacy. 'Enactive mastery' pertains to the experience of actual accomplishment and the subsequent increase in confidence that results. 'Verbal persuasion' is the notion that other people can give encouragement or otherwise make the case for increased competence. 'Vicarious experience' refers to the increase in confidence that sometimes results when people see a comparable other person coping successfully in a given situation. Finally, Bandura included 'physiological state' as a source of self-efficacy, reasoning that the experience of being less provoked than expected should increase confidence in a stressful circumstance.

Evidence suggests that psychosocial determinants help mitigate the effect of self-efficacy on health outcomes $[7,8]$. Relevant psychosocial determinants in regards to

\section{Biomed Central}


the efficacy/health relationship include perceived stress, sense of control, social support and perceived standing in society [9], while fatalism is a key component of Bandura's social cognitive theory relating to selfefficacy and health [10].

Although low parental self-efficacy has been associated with adverse dental outcomes among children, there has been little documented evidence of the role of selfefficacy in adult oral health outcomes. Even less has been reported on groups known to be at high risk of dental disease. Aboriginal Australians [11] and pregnant women [12] are both groups at risk of dental disease. The purpose of this investigation was to determine if low self-efficacy was associated with poor self-rated oral health, a recognised proxy marker of both clinical oral health status [13] and oral health-related quality of life [14], among a convenience sample of Australian women pregnant with an Aboriginal child.

\section{Methods}

\section{Study data and design}

Participants were 446 women pregnant with an Aboriginal child in South Australia, Australia, who were part of a randomised controlled trial involving prevention of early childhood caries. Data used in this paper were thus cross-sectional and from a convenience sample. The participation rate was 100 percent and there were no incomplete responses (due to questionnaire data being collected via interview. Written informed consent was received). Participants represented around one-third of those who were eligible for the study during the study period (Feb 2011 to May 2012). We were unable to ascertain if our participants differed in significant ways to the source population due to the lack of Census, or other data, specifically pertaining to women pregnant with Aboriginal children. Participants were recruited from a range of sources including referrals from Aboriginal groups, community services and hospitals. The study received approval from the University of Adelaide Human Research Ethics Committee, the Aboriginal Health Council of South Australia, the Government of South Australia and the Human Research Ethics Committees of participating South Australian hospitals.

\section{Dependent variable}

Poor self-rated oral health was defined as a response of 'fair' or 'poor' to the question: 'How do you think your dental health is?' Other options included 'excellent', 'very good' or 'good'.

\section{Independent variables}

Self-efficacy was based on an instrument developed by Finlayson and colleagues [15]. It was measured using a 6item scale, asking participants to indicate how confident they feel about their ability to brush their teeth at night when they were: (1) under a lot of stress; (2) depressed; (3) anxious; (4) feeling that they were too busy; (5) tired or; (6) worried about other things in their life. The four response options ranged from 'very confident' to 'not at all confident'. The possible score range is 0 to 24 , with high scores indicating high self-efficacy. Alpha was 0.91. Self-efficacy was dichotomised based on a median split, with low self-efficacy pertaining to scores of 0 to 11 and high self-efficacy pertaining to scores of $12+$.

The socio-demographic factors included age, education, income and ownership of a means-tested Government Health Care Card. A means-tested Government Health Care Card is provided to individuals on low income in Australia and enables them to have cheaper health care services and less expensive medicines. The age range of the sample was 14 to 43 years (mean 24.9 years, sd $=5.9$ ). Age was dichotomised into ' 14 to 24 years' and ' 25 yearst'. Education was dichotomized into 'high school or less' or 'trade/technical or University', while Income was dichotomized into 'Job' or 'Centrelink' (welfare). 'Centrelink' is the Australian agency which provides welfare payments to those who are unemployed. Because such a small proportion of respondents earned their income from non-welfare means, the 'job' category included all forms of paid employment.

The psychosocial-related factors included stress, control, social support and subjective social standing. Stress was measured by the Perceived Stress Scale [16], which evaluates the frequency that people appraise situations as threatening and their appraised capacity to cope with threatening situations. There are 14 items in total, with five response options ranging from 'not at all' to 'very often'. The possible score range is 0 to 56 , with high scores indicating high stress. Alpha was 0.75 . Stress was dichotomised, with low stress reflecting scores of 0 to 27 and high stress reflecting scores of $28+$.

Control was assessed by the Sense of Personal Control scale [17], which comprises two dimensions referred to as 'personal mastery' and 'perceived constraint'. The five response options for the 12 items range from 'strongly disagree' to 'strongly agree', with high scores indicating high control. The possible score range is 0 to 48 and alpha was 0.83 . Control was dichotomised, with low control reflecting scores of 0 to 34 and high control reflecting scores of $35+$.

Social support was assessed by four items, each designed to evaluate one of four dimensions of social support as theorised by House [18]. The dimensions include emotional, appraisal, instrumental and informational support. The five response options include 'strongly agree' to 'strongly disagree', with high scores indicating high social support. The possible score range is 0 to 16 (high scores equal high social support) and alpha was 
0.86. Social support was dichotomised into 0 to 13 for low social support and 14+ for high social support.

Subjective Social Standing was assessed by the MacArthur Scale of Subjective Social Status [19]. The scale consists of a 10-rung ladder visual analogue scale on which participants rank themselves relative to others in their community ('place in society'). Mean scores are calculated, with the possible range being 0 to 10 . Subjective Social Standing was dichotomised, with low subjective social standing categorised as 0 to 5 and high subjective social support categorised as $6+$.

The social cognitive factor included oral health-related fatalism and was based on an instrument developed by Finlayson and colleagues [15]. It was measured using a 3-item scale, asking participants to indicate their level of agreement with three oral health scenerios including pain, tooth loss and child dental caries. The five response options ranged from 'strongly disagree' to 'strongly agree'. The possible score range is 0 to 12 , with high scores indicating high fatalism. Alpha was 0.83 . Fatalism was dichotomised, with low fatalism pertaining to scores of 0 to 8 and high fatalism pertaining to scores of $9+$.

The risk behaviour included the question 'did you brush your teeth yesterday?', with response options including 'yes' or 'no'.

\section{Analytic methods}

Complete data were available for 446 participants. Correlation tests confirmed the existence of weak associations among the primary exposure (self-efficacy) and covariates (Pearson's correlation coefficient range $0.1-0.4)$. No variables needed to be excluded due to collinearity. In bivariate analyses, prevalences and corresponding confidence intervals and p-values were generated by the 'cross-tabulations' analytical approach in SPSS. Blocks of explanatory variables were entered into a binary logistic regression model in six steps, as predicated by our conceptual model
(Figure 1). The dependent variable of these models was self-rated oral health 'fair' or 'poor'. Self-efficacy was entered in Model 1, with the main effect presented as a prevalence odds ratio (POR) with its 95\% confidence interval $(95 \% \mathrm{CI})$. The four socio-demographic factors were entered into Model 2, four psychosocial factors entered into Model 3, fatalism entered into Model 4 and the risk behavior entered into Model 5. The final model (Model 6) comprised all factors. This order of modelbuilding was chosen so that individual effects of the domains represented in the conceptual model (sociodemographic factors, psychosocial factors, social cognitive factors and risk behaviours) could be assessed in relation to the outcome measure (poor self-rated oral health) before all variables were analysed in the final model. It is important to note that the final model was built based on apriori selection of covariates according to the conceptual model (Figure 1) as opposed to covariate selection based upon bivariate statistics. The degree of attenuation was calculated by the $1-(\ln ($ adjusted OR $) / \ln$ (unadjusted OR) formula. (Brotman [20]). We need to be clear that these estimates do not reflect risk. Data were analysed using IBM SPSS Statistics version 20.

\section{Results}

Just over half the participants (55 percent) rated their oral health as 'fair' or 'poor' (Table 1). Almost two-thirds (63 percent) were categorized as having 'low' selfefficacy. Just over half (52 percent) the participants were aged 14 to 24 years, 72 percent had achieved high school or less as their highest education attainment, 86 percent had welfare-based income and 82 percent owned a health care card. Over one-third (37 percent) were categorized as having high stress, while 62 percent had a low sense of control. Around 58 percent reported low social support and 56 percent reported low subjective social standing. Around 39 percent of participants reported

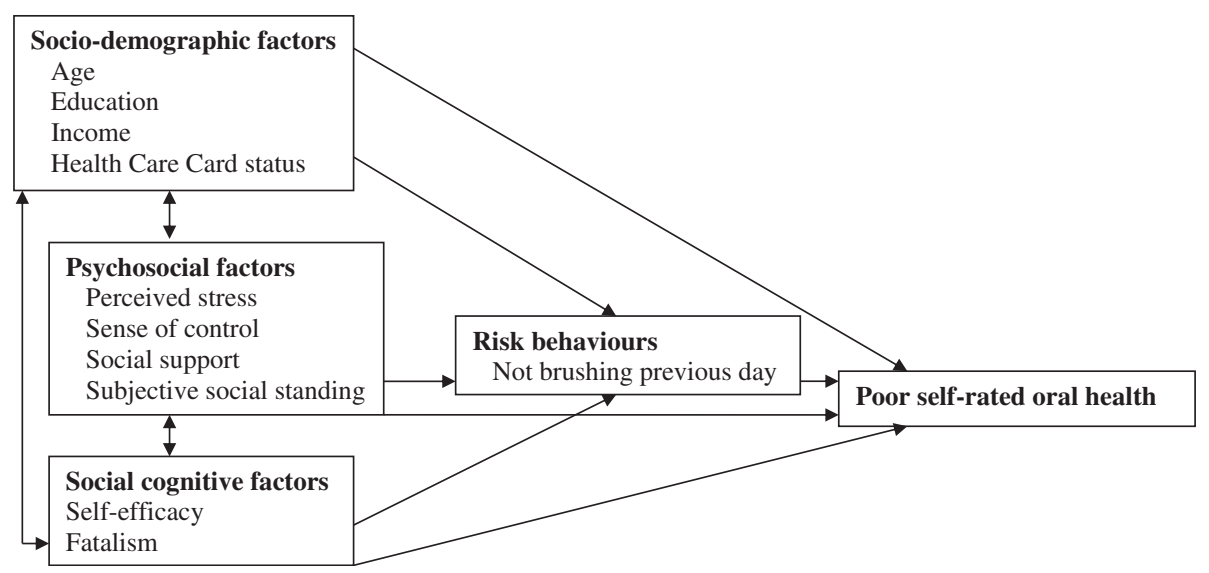

Figure 1 Postulated low self-efficacy and poor self-rated oral health pathway for women expecting Aboriginal babies. 
Table 1 Frequencies, prevalences and unadjusted odds ratios for low self-efficacy and poor self-rated oral health

\begin{tabular}{|c|c|c|c|c|c|}
\hline & $\begin{array}{l}\text { Frequency } \\
(95 \% \mathrm{Cl})\end{array}$ & $\begin{array}{l}\text { Prevalence low } \\
\text { self-efficacy }(95 \% \mathrm{Cl})\end{array}$ & $\begin{array}{l}\text { Unadjusted odds } \\
\text { ratio }(95 \% \mathrm{Cl})\end{array}$ & $\begin{array}{l}\text { Prevalence poor self-rated } \\
\text { oral health }(95 \% \mathrm{Cl})\end{array}$ & $\begin{array}{l}\text { Unadjusted odds } \\
\text { ratio }(95 \% \mathrm{Cl})\end{array}$ \\
\hline \multicolumn{6}{|l|}{ Self-rated oral health } \\
\hline Excellent, very good or good & $45.7(41.1-50.4)$ & $52.2(44.0-60.0)^{*}$ & $0.42(0.27-0.65)^{*}$ & - & - \\
\hline Fair or poor (ref) & $54.3(49.6-58.9)$ & $72.4(65.6-78.3)$ & 1.00 & - & - \\
\hline \multicolumn{6}{|l|}{ Self-efficacy } \\
\hline Low (0-11) & $63.3(58.1-68.2)$ & - & - & $62.9(56.3-69.0)^{*}$ & $2.40(1.54-3.74)^{*}$ \\
\hline High (12+; ref) & $36.7(31.8-41.9)$ & - & - & $41.4(33.2-50.1)$ & 1.00 \\
\hline \multicolumn{6}{|l|}{ Age } \\
\hline 14 to 24 years & $52.2(47.5-57.0)$ & $60.8(53.2-68.0)$ & $0.80(0.51-1.25)$ & $50.7(44.1-57.2)$ & $0.76(0.52-1.12)$ \\
\hline $25+$ years (ref) & $47.8(43.0-52.5)$ & $66.1(58.5-72.9)$ & 1.00 & $57.4(50.5-64.1)$ & 1.00 \\
\hline \multicolumn{6}{|l|}{ Education } \\
\hline High school or less & $71.6(67.2-75.6)$ & $65.3(59.2-71.0)$ & $1.41(0.87-2.27)$ & $56.5(50.9-61.8)^{*}$ & $1.43(0.94-2.16)$ \\
\hline Trade or University (ref) & $28.4(24.4-32.8)$ & $57.3(47.2-66.8)$ & 1.00 & $47.6(39.0-56.4)$ & 1.00 \\
\hline \multicolumn{6}{|l|}{ Income } \\
\hline Job & $14.1(11.1-17.6)$ & $68.0(53.9-79.4)$ & $1.28(0.68-2.44)$ & $43.5(31.8-56.1)$ & $0.61(0.35-1.04)$ \\
\hline Centrelink (ref) & $85.9(82.4-88.9)$ & $62.3(56.6-67.6)$ & 1.00 & $55.9(50.9-60.9)$ & 1.00 \\
\hline \multicolumn{6}{|l|}{ HCC status } \\
\hline Yes & $82.2(78.3-85.6)$ & $62.9(57.1-68.4)$ & $0.85(0.47-1.54)$ & $55.9(50.7-61.0)$ & $1.52(0.93-2.50)$ \\
\hline No & $17.8(14.4-21.7)$ & $66.7(53.9-77.4)$ & 1.00 & $45.5(34.7-56.7)$ & 1.00 \\
\hline \multicolumn{6}{|l|}{ Perceived stress } \\
\hline Low (0-27) & $63.4(58.8-67.9)$ & $53.9(47.0-60.6)^{*}$ & $0.39(0.24-0.62)^{*}$ & $47.8(41.9-53.8)^{*}$ & $0.49(0.33-0.73)^{*}$ \\
\hline High (28+) & $36.6(32.1-41.2)$ & $75.2(67.1-81.8)$ & 1.00 & $65.2(57.4-72.2)$ & 1.00 \\
\hline \multicolumn{6}{|l|}{ Sense of control } \\
\hline Low (0-34) & $62.1(57.4-66.5)$ & $72.7(66.3-78.2)^{*}$ & $3.13(1.96-5.00)^{*}$ & $59.6(53.6-65.3)^{*}$ & $1.73(1.17-2.56)^{*}$ \\
\hline High (35+) & $37.9(33.5-42.6)$ & $46.0(37.4-54.8)$ & 1.00 & $46.1(38.6-53.7)$ & 1.00 \\
\hline \multicolumn{6}{|l|}{ Social support } \\
\hline Low (0-13) & $58.3(53.7-62.9)$ & $69.3(62.8-75.2)^{*}$ & $1.96(1.25-3.03)^{*}$ & $59.8(53.7-65.7)^{*}$ & $1.72(1.17-2.51)^{*}$ \\
\hline High (14+) & $41.7(37.1-46.3)$ & $53.7(45.2-61.9)$ & 1.00 & $46.5(39.4-53.7)$ & 1.00 \\
\hline \multicolumn{6}{|l|}{ Subjective social standing } \\
\hline Low (0-5) & $55.7(50.9-60.3)$ & $72.2(65.4-78.0)^{*}$ & $2.56(1.61-4.00)^{*}$ & $61.7(55.3-67.6)^{*}$ & $1.84(1.25-2.71)^{*}$ \\
\hline High (6+) & $44.3(39.7-49.1)$ & $50.3(42.2-58.4)$ & 1.00 & $46.6(39.6-53.7)$ & 1.00 \\
\hline \multicolumn{6}{|l|}{ Fatalism } \\
\hline Low (0-8) & $61.5(56.9-65.9)$ & $61.6(54.9-67.8)$ & $0.82(0.52-1.28)$ & $54.6(48.6-60.4)$ & $1.01(0.69-1.48)$ \\
\hline High (9+) & $38.5(34.1-43.1)$ & $66.2(57.7-73.7)$ & 1.00 & $54.4(46.8-61.7)$ & 1.00 \\
\hline \multicolumn{6}{|l|}{ Brush yesterday } \\
\hline Yes & $75.1(70.8-79.0)$ & $55.5(49.3-61.5)^{*}$ & $0.22(0.12-0.43)^{*}$ & $47.8(42.2-53.3)^{*}$ & $0.45(0.29-0.72)^{*}$ \\
\hline No (ref) & $24.9(21.0-29.2)$ & $84.8(75.1-91.2)$ & 1.00 & $67.0(57.5-75.3)$ & 1.00 \\
\hline
\end{tabular}

high levels of fatalism and one quarter (25 percent) had not brushed their teeth the previous day. A higher proportion of participants who reported low self-efficacy had lower levels of self-rated oral health, higher stress, lower sense of control, lower perceived social support, lower subjective social standing and did not brush the previous day (Table 1). Fair or poor self-rated oral health was associated with low self-efficacy, high school or less education attainment, high stress, low sense of control, low social support, low subjective social standing and not brushing the previous day.

In an unadjusted multivariable model, those with low self-efficacy had nearly two and a half times the odds of rating their oral health as 'fair' or 'poor' (Table 2, Model 1). 
Table 2 Multivariable models evaluating risk indicators for poor self-rated oral health among Australians expecting an Aboriginal child

\begin{tabular}{|c|c|c|c|c|c|c|}
\hline & $\begin{array}{l}\text { Model } 1 \\
\text { (POR, 95\% Cl) } \\
\end{array}$ & $\begin{array}{l}\text { Model } 2 \\
\text { (POR, 95\% Cl) }\end{array}$ & $\begin{array}{l}\text { Model } 3 \\
(\text { POR, 95\% Cl) }\end{array}$ & $\begin{array}{l}\text { Model } 4 \\
(\text { POR, 95\% Cl) } \\
\end{array}$ & $\begin{array}{l}\text { Model } 5 \\
(\text { POR, 95\% Cl) }\end{array}$ & $\begin{array}{l}\text { Model } 6 \\
(\text { POR, 95\% Cl) } \\
\end{array}$ \\
\hline \multicolumn{7}{|l|}{ Self-efficacy } \\
\hline Low (0-11) & $2.40(1.54-3.74)^{*}$ & $2.19(1.37-3.51)^{*}$ & $2.07(1.28-3.36)^{*}$ & $2.42(1.55-3.78)^{*}$ & $2.11(1.32-3.36)^{*}$ & $1.80(1.05-3.08)^{*}$ \\
\hline High (12+; ref) & 1.00 & 1.00 & 1.00 & 1.00 & 1.00 & 1.00 \\
\hline \multicolumn{7}{|l|}{ Age } \\
\hline 14 to 24 years & - & $0.62(0.39-0.98)^{*}$ & - & - & - & $0.62(0.38-1.03)$ \\
\hline $25+$ years (ref) & - & 1.00 & - & - & - & 1.00 \\
\hline \multicolumn{7}{|l|}{ Education } \\
\hline High school or less & - & $1.23(0.73-2.07)$ & - & - & - & $1.36(0.79-2.36)$ \\
\hline Trade or University (ref) & - & 1.00 & - & - & - & 1.00 \\
\hline \multicolumn{7}{|l|}{ Income } \\
\hline Job & - & $0.77(0.33-1.76)$ & - & - & - & $1.00(0.41-2.42)$ \\
\hline Centrelink (ref) & - & 1.00 & - & - & - & 1.00 \\
\hline \multicolumn{7}{|l|}{ HCC status } \\
\hline Yes & - & $1.04(0.48-2.23)$ & - & - & - & $1.08(0.48-2.43)$ \\
\hline No & - & 1.00 & - & - & - & 1.00 \\
\hline \multicolumn{7}{|l|}{ Perceived stress } \\
\hline Low (0-27) & - & - & $0.77(0.46-1.29)$ & - & - & $0.80(0.46-1.40)$ \\
\hline High (28+) & - & - & 1.00 & - & - & 1.00 \\
\hline \multicolumn{7}{|l|}{ Sense of control } \\
\hline Low (0-34) & - & - & $0.99(0.57-1.70)$ & - & - & $1.13(0.63-2.06)$ \\
\hline High (35+) & - & - & 1.00 & - & - & 1.00 \\
\hline \multicolumn{7}{|l|}{ Social support } \\
\hline Low (0-13) & - & - & $1.35(0.82-2.23)$ & - & - & $1.33(0.78-2.26)$ \\
\hline High (14+) & - & - & 1.00 & - & - & 1.00 \\
\hline \multicolumn{7}{|l|}{ Subjective social standing } \\
\hline Low (0-5) & - & - & $1.34(0.83-2.18)$ & - & - & $1.12(0.66-1.91)$ \\
\hline High (6+) & - & - & 1.00 & - & - & 1.00 \\
\hline \multicolumn{7}{|l|}{ Fatalism } \\
\hline Low (0-8) & - & - & - & $1.17(0.75-1.82)$ & - & $0.95(0.56-1.60)$ \\
\hline High (9+) & - & - & - & 1.00 & - & 1.00 \\
\hline \multicolumn{7}{|l|}{ Brush yesterday } \\
\hline Yes & - & - & - & - & $0.74(0.43-1.27)$ & $0.88(0.48-1.62)$ \\
\hline No (ref) & - & - & - & - & 1.00 & 1.00 \\
\hline-2 Log Liklihood & 465.1 & 425.3 & 423.0 & 464.7 & 448.7 & 374.2 \\
\hline Nagelkerke $\mathrm{R}^{2}$ & 0.057 & 0.068 & 0.081 & 0.059 & 0.054 & 0.088 \\
\hline
\end{tabular}

Addition of socio-demographic factors to the selfefficacy variable attenuated the effect of low selfefficacy on poor self-rated oral health by 10 percent (Table 2, Model 2). Addition of the psychosocial factors to the self-efficacy variable attenuated the odds by 17 percent (Table 2, Model 3), while addition of the social cognitive variable fatalism to the self-efficacy variable increased the odds by 1 percent (Table 2, Model 4). Inclusion of the behavioural risk factor 'not brushing previous day' to the self-efficacy variable attenuated the odds by 15 percent (Table 2, Model 5). Low self-efficacy persisted as a risk indicator for poor self-rated oral health in the final model, which included all covariates. In this final model, the odds were attenuated by 32 percent (Table 2, Model 6). 


\section{Discussion}

Low self-efficacy was a risk indicator for poor self-rated oral health in this convenience sample of pregnant Aboriginal Australians; a group recognised as being of high risk of both dental caries and periodontal disease [11]. This association persisted even after adjusting for sociodemographic, psychosocial, social cognitive and behaviour covariates. In the final model, self-efficacy was the only variable that remained significant. To the best of our knowledge, this is the first time risk indicators for self-rated oral health in an Australian Aboriginal population have been reported and the first time an association between self-efficacy and self-rated oral health in any population has been established.

Due to the cross-sectional nature of our study, the findings cannot be considered to be causal. Indeed, it is possible that poor self-rated oral health may lead to feelings of low self-efficacy. The self-report nature of the data may have led to an under-estimation of these factors, with incorrect responses potentially given for any number of reasons (social desirability bias, difficulty understanding English, not comprehending question). However, we took great care with interviewing and, in any case, non-differential under-reporting would have resulted in more conservative estimates, meaning our findings are unlikely to be spurious. Evidence from the literature suggests, however, that selfefficacy is likely to be a driver of certain behaviours that lead to health outcomes, with clinical studies typically using self-efficacy as an antecedent to behaviour modification. In the dental field, McCaul and colleagues [21] reported that self-efficacy was significantly related to both retrospective and prospective selfmonitored frequency of brushing and flossing among college students, while Tedesco et al. [22] found that linking self-efficacy variables to theory of reasoned action variables significantly increased the variance observed in brushing and flossing behaviours. In a study that compared psychological characteristics such as self-efficacy, locus of control and self-esteem in relation to oral health habits, dental caries and periodontal disease, Syrjala et al. [23] reported that only selfefficacy was associated with all dental outcomes. In regards to self-efficacy-based oral health interventions, Kakudate and colleagues [24] conducted a randomised controlled trial to compare effectiveness of an oral hygiene-based enhanced self-efficacy intervention with conventional oral hygiene instruction. The intervention group had improved plaque index scores, toothbrushing duration and frequency of inter-dental cleaning in comparison with the control group, leading the authors to conclude that the effectiveness of a behavioural intervention to enhance self-efficacy and promote oral health-related behavioural change was observed.

\section{Conclusion}

The findings suggest that, in our study, the self-efficacy association with poor self-reported oral health exists beyond the tooth-brushing pathway. Other potential mechanisms linking self-efficacy and oral health status may include high-sugar diet, problem-based dental attendance or dental fear. Future studies would do well to include analyses of these additional factors.

Self-efficacy among Aboriginal Australians has not been extensively researched. However, the Australian Institute of Health and Welfare, who are involved in the conduct and publication of Australia's National Aboriginal and Torres Strait Islander Health Surveys, suggests that social and emotional well-being concepts such as self-efficacy should be considered in future waves [25]. Our findings give some evidence that the role of self-efficacy in health outcomes such as selfrated oral health among Aboriginal populations may be under-appreciated, and that this association needs to be further explored. The convenience sample means the findings are not able to be generalised to other population groups, meaning further research among other populations, preferably utilising a longitudinal design, is required.

\section{Competing interest}

The authors declare that they have no competing interests.

\section{Authors' contributions}

LMJ conceived of the study, participated in its design and co-ordination and drafted the manuscript. EJP made substantial contributions to the design of the study and interpretation of data. KRT made substantial contributions to the design of the study and interpretation of data. JSB was involved in drafting the manuscript or revising it critically for important intellectual content. HPL was involved in drafting the manuscript or revising it critically for important intellectual content. All authors read and approved the final manuscript.

\section{Acknowledgement}

The authors gratefully acknowledge the support of Baby Teeth Talk (Australia) study participants, study staff and partners: South Australian Dental Service, Colgate Palmolive, Women's and Children's Hospital, Lyell McEwen Hospital, Flinders' Medical Centre, Aboriginal Family Support Services, Aboriginal Primary Health Unit, Metro Aboriginal Family Birthing Program, Kura Yerlo Centre, Aboriginal Legal Rights Movement, Wodlitinattoai Program, Ninko Kutangga Patpangga, Kanggawodli Step Down Service, Kaurna Plains, Fleurieu Families, Gilles Plains Community Health, MADEC Community Support Services, Naporendi Aboriginal Forum, Nunga MiMinar, Aboriginal Strategy Unit of Families South Australia, Inbarendi College, Para West Adult Campus, and the Muno Parra Medical Centre, Second Story, Inner Southern Health Service, The Corner House, Louise Place, PARKS, Talking Realities Program, Child Youth Women's Health Services, Southern Junction Community Services, TAFE campuses, GP Plus centres, employment programs, childcare centres, women's centres, domestic violence shelters, and primary and high schools. This study was funded by the National Health and Medical Research Council of Australia (NHMRC, Project Grant 627350). LMJ is supported by NHMRC Career Development Fellowship \#1045800.

\section{Author details}

${ }^{1}$ Australian Research Centre for Population Oral Health, University of Adelaide, Adelaide, SA 5005, Australia. ${ }^{2}$ Otago University School of Dentistry, Dunedin, New Zealand. ${ }^{3}$ Toronto University School of Dentistry, Toronto, ON, Canada. 
Received: 18 December 2013 Accepted: 18 March 2014

Published: 2 April 2014

\section{References}

1. Bandura A: Self-efficacy: toward a unifying theory of behaviour change. Psychol Rev 1977, 84:191-215.

2. Klepac RK, Dowling J, Hauge G: Characteristics of clients seeking therapy for the reduction of dental avoidance: Reactions to pain. J Behau Ther Exp Psych 1982, 13:293-300.

3. Kent G, Gibbons R: Self-efficacy and the control of anxious cognitions. J Behav Ther Exp Psych 1987, 18:33-40.

4. Reisine S, Litt M: Social and psychological theories and their use for dental practice. Int Dent J 1993, 43:279-287.

5. Pine CM, Adair PM, Petersen PE, Douglass C, Burnside G, Nicoll AD, Gillett A, Anderson R, Beighton D, Jin-You B, Broukal Z, Brown JP, Chestnutt IG, Declerck D, Devine D, Espelid I, Falcolini G, Ping FX, Freeman R, Gibbons D, Gugushe T, Harris R, Kirkham J, Lo EC, Marsh P, Maupomé G, Naidoo S, Ramos-Gomez F, Sutton BK, Williams S: Developing explanatory models of health inequalities in childhood dental caries. Community Dent Health 2004, 21:86-95.

6. Finlayson TL, Siefert K, Ismail Al, Sohn W: Maternal self-efficacy and 1-5-year-old children's brushing habits. Community Dent Oral Epidemiol 2007, 35:272-281.

7. Lee J, Donlan W, Cardoso EE, Paz JJ: Cultural and social determinants of health among indigenous Mexican migrants in the United States. Soc Work Public Health 2013, 28:607-618.

8. Wang J, Sereika SM, Styn MA, Burke LE: Factors associated with healthrelated quality of life among overweight or obese adults. J Clin Nurs 2013, 22:2172-2182.

9. Bailey A, Sharma M, Jubin M: The mediating role of social support, cognitive appraisal, and quality health care in black mothers' stress-resilience process following loss to gun violence. Violence Vict 2013, 28:233-247.

10. Lagos VI, Perez MA, Ricker CN, Blazer KR, Santiago NM, Feldman N, Viveros L, Weitzel JN: Social-cognitive aspects of underserved Latinas preparing to undergo genetic cancer risk assessment for hereditary breast and ovarian cancer. Psychooncology 2008, 17:774-782.

11. Roberts-Thomson KF, Spencer AJ, Jamieson LM: Oral health of aboriginal and Torres strait islander Australians. Med J Aust 2008, 188:592-593.

12. US Department of Health and Human Services: Oral health in America: a report of the surgeon general. Rockville (MD): U.S. Department of Health and Human Services, National Institute of Dental and Craniofacial Research, National Institutes of Health; 2000.

13. Zaitsu T, Ueno M, Shinada K, Ohara S, Wright FA, Kawaguchi Y: Association of clinical oral health status with self-rated oral health and GOHAI in Japanese adults. Community Dent Health 2011, 28:297-300.

14. Sischo L, Broder HL: Oral health-related quality of life: what, why, how, and future implications. J Dent Res 2011, 90:1264-1270.

15. Finlayson TL, Siefert K, Ismail Al, Delva J, Sohn W: Reliability and validity of brief measures of oral health-related knowledge, fatalism, and selfefficacy in mothers of African American children. Pediatr Dent 2005, 27:422-428.

16. Cohen S, Kamack T, Mermelstein R: A global measure of perceived stress. $J$ Health Soc Behav 1983, 24:385-396.

17. Lachman ME, Weaver SL: The sense of control as a moderator of social class differences in health and well-being. J Pers Soc Psycho/ 1998, 74:763-773.

18. House JS: Work Stress and Social Support. New York: Random House; 1981.

19. Adler NE, Epel ES, Castellazzo G, Ickovics JR: Relationship of subjective and objective social status with psychological and physiological functioning. Health Psychol 2000, 19:586-592.

20. Brotman DJ: Mediators of the association between mortality risk and socioeconomic status. JAMA 2006, 296:763-764.

21. McCaul KD, Glasgow RE, Gustafson C: Predicting levels of preventive dental behaviors. J Am Dent Assoc 1985, 111:601-605.

22. Tedesco LA, Keffer MA, Davis EL: Effect of a social cognitive intervention on oral health status, behaviour reports, and cognitions. J Periodontol 1992, 63:567-575.

23. Syrjala AM, Ylostalo P, Niskanen MC, Knuuttila ML: Relation of different measures of psychological characteristics to oral health habits, diabetes adherence and related clinical variables among diabetic patients. Eur J Oral Sci 2004, 112:109-114.

24. Kakudate N, Morita M, Sugai M, Kawanami M: Systematic cognitive behavioral approach for oral hygiene instruction: a short-term study. Patient Educ Couns 2009, 74:191-196.

25. Australian Institute of Health and Welfare: Measuring the social and emotional wellbeing of Aboriginal and Torres Strait Islander peoples. Canberra: Australian Institute of Health and Welfare; 2009.

doi:10.1186/1472-6831-14-29

Cite this article as: Jamieson et al:: Self-efficacy and self-rated oral health among pregnant aboriginal Australian women. BMC Oral Health

$201414: 29$.

\section{Submit your next manuscript to BioMed Central and take full advantage of:}

- Convenient online submission

- Thorough peer review

- No space constraints or color figure charges

- Immediate publication on acceptance

- Inclusion in PubMed, CAS, Scopus and Google Scholar

- Research which is freely available for redistribution

Submit your manuscript at www.biomedcentral.com/submit
C Biomed Central 